FRACTURE OF THE SKULL AT ITS BASE, \&c.

\section{To the Editor of The LanceT.}

SiR:-I shall feel greatly obliged by your inserting the following case of extensive fracture of the skull, in your widely circulated Journal. I am, Sir, your obedient servant,

Northampton Infirmary,

H. G. Bull. Oct. 2, 1837 .

Richard Steele, retat. 35, porter, was admitted, August 22, with compound fracture of the right leg, about three inches above the anklejoint. He had also a slight scalp wound at the superior part of the occipital bone, but no fracture could be discovered. The accident occurred from the shafts of the cart on which he was riding, breaking as he, was going down a hill, when he fell upon his head, being at the time in a state of intoxication. He has been in the habit of drinking to excess daily, for the last year or two.

The accident happened about nine o'clock on the evening of the 21 st, but he did not reach the hospital until four o'clock on the following afternoon. He has been insensible ever since the accident happened, and on his admittance he was so restless as to require a strait-waistcoat. On the morning previous to his admission, he had vomited a large quantity of blood, for which he was bled by a medical man residing at the place where the accident happened. At the time of his admission, his pulse was 72 , full and strong; pupils dilated; bowels have not been moved since the accident. It was at first supposed that he was labouring under delirium tremens from drinking. The following medicines were prescribed.

Tincture of opium, 50 minims.

Camphor mixture, $3 \mathrm{xj}$. Make a dranght, to be taken immediately.

A common enema.

Camphor mixture; saline; of each, $3 \mathrm{vj}$.

Sulph. of magnesia, $3 \mathbf{j}$. Make a draught, to be taken in four hours. Shave the head, and afterwards apply a cold lotion to the scalp. Had great difficulty in swallowing the medicines.

23rd. Nine, A. M. Pulse 120, wiry, and intermittent. The enema has operated several times. Continue the medicines.

Four, P.M. Has not been able to take his medicine since the morning, and is evidently sinking fast.

24th. Died early in the morning.

Sect. Cadaveris, twenty-four hours after death.-On removing the calvarium, two large clots of coagulated venous blood were found on the anterior superior part of each hemisphere, and another between them lying

on the corpus callosum. On removing the brain, a fracture at the base of the skull was discovered commencing near the tuberosity of the occiput, and extending parallel to its spine down to the foramen magnum. Another fracture encircled the foramen mag. num, commencing at the: above ; it passed through the petrous portion of the temporal bone, on each side of the sella turcica, where it met its fellow. The posterior clinoid processes were nearly broken off. The fracture then passed forwards as far as the cribriform plate of the ethmoid bone. The other parts of the body were not examined.

*** Although from the extensive injury of the base of the skull, discovererl after death, medical treatment could not have been of any avail in the case described by Mr. Bull, we cannot but think that the treatment presents an example of carelessness such as is more frequently witnessed in some of our London hospitals, than any where else among educated surgeons. A man falls upon his head, is brought to an hospital, with a wound of the scalp, in a state of insensibility, and evidently labour. ing under symptoms of effusion into, and laceration of, the brain, and yet the bones of the cranium are not examined! The man is supposed to labour under delirium tremens, and the short space of time which, under different circumstances, might have been vitally important to the patient, is occupied in the administration of opiates and insignificant draughts.

Our correspondent has omitted to mention the person by whom this case was treated. The practice is very like that of some protege of the Poor-Law Commis. sioners.

\title{
PIGMENT OF ANTIMONY.
}

\section{To the Editor of The LANCET.}

SIR :-In a report of the proceedings of the British Association, I observe a paper, by Dr. Traill, "On a New Compound of Antimony as a Pigment," lately discovered by him. Permit me, through the medium of your valuable Journal, to inform the Doctor that the compound in question was discovered previous to his communication being made. I have prepared it myself repeatedly, and have used it as a blue writing ink, the pigment being suspended in a solution of isinglass. As a proof of my assertion I can show specimens of it in my day-book and ledger, written nearly two years ago. I am, Sir, your obedient servant,

25, Princes-street,

Old Compton-street, Soho, Oct. 5, 1837.
J. C.Apprson, Chemist, 